\title{
The Mainline of Organizational Behavior Management: Binary Nonequilibrium
}

\author{
Jie ZHEN* \\ East China University of Political Science and Law, Shanghai, China \\ jetzhen@163.com
}

Keywords: Organizational behavior management, Binary Nonequilibrium, Mainline.

\begin{abstract}
The binary nonequilibrium is the mainline of organizational behavior management. The so-called binary refers to the hard factors and soft ones that affect the behavior of the organization and can be subclassified based on individual-level, group-level and organization-level. While the nonequilibrium means the two types of factors have different degree of importance at the various development stages of the organization. Under the guidance of the mainline, behavioral structure is generated and constituted of behavioral identification, selection and feedback within the specific scope of organizational behavior, which determines the relevant behavioral process. Finally, the behavioral performance is attained and the paradigm of the organizational behavior management is formed.
\end{abstract}

\section{Introduction}

The research on the management of organizational behavior attempts to constitute a reasonable and clear organizational behavior system taking individual-level behavior, group-level behavior and organization-level behavior as the main body and taking the internal and external behavior of the organization as the framework. The three levels and the scope of organizational behavior gradually extending from inside to outside follow the logic system of binary nonequilibrium which combines the hard factors and soft ones. Binary nonequilibrium means that the organizational behavior is the result of the binary elements including hard factors and soft ones at the same time while the degree of importance of the two types of factors is disequilibrium at different time periods. The binary disequilibrium, as an active state of the two types of factors in their mutual evolution process during different stages, embodies the essential characteristics of utility and evolution process of organizational behavior. Individual behavior, group behavior and organizational behavior are all influenced by the combined action of the operational soft factors and the basic hard factors. Moreover, the importance of binary elements will present nonequilibrium evolvement rule which is from appreciating soft factors while despising hard factors to emphasizing hard factors while ignoring soft factors and to attach equal importance to hard and soft factors with the enlargement of the scale of the organization.

\section{The Thought Mainline of Organization Behavior}

The content of organizational behavior forms the mainline of thought from the perspective of duality (see figure 1). The hard factor here refers to the influence factors that play the role of platform, carrier or fundamental function in various levels. The soft factor exerts an effect on the behaviors of various levels centering on the fundamental role of the hard factor. To be specific, individual-level behavior is launched according to the logic thinking of individual motivation, perception, capability and personality. Among them, the capability belongs to hard factor affecting individual behavior while motivation, perception and personality belong to the soft factors that affect individual behavior. Group-level behavior is launched following the logic thinking of leadership, decision making, communication and conflict. Among them, leadership belongs to hard factor affecting the group behavior while decision making, communication and conflict belong to 
the soft factors that affect group behavior. Organization-level behavior is launched following the logic thinking of structure, culture, ethics and charity, ${ }^{[1]}$ revolution. Among them, the structure belongs to hard factor influencing organizational behavior, and culture, ethics and charity, revolution belong to the soft factors that influence organizational behavior. It can be seen that in these three levels, the behavior of each level is influenced by both soft factors and hard factors.

At the early stage of organizational development, the soft factors at all levels play an important role while the corresponding hard factors have not reached a high level. For example, in this stage, individuals have strong motivation, but their abilities are not enough to meet the needs of the organization; the group emphasizes the effect of decision-making and the communication and the resolution of the conflicts, but the leadership level of the group needs to be improved; at the organizational level, cultural matching and transformational effect are emphasized, but the structure is not very standardized. In the intermediate stage of organization development, all the three levels start to focus on the development of hard factors on the basis of the soft factors which have been strengthened during the early stages. The work in the process should emphasize the improvement of individuals and group leadership and the optimizing of organizational structure through the gradual maturity of the concrete mechanisms. In the last stage of organizational development, both the soft factors and the hard factors reach a higher level. The two types of the factors complete a state of equilibrium due to the need of development. From the perspective of the whole development cycle, the development of soft factors and hard factors is unbalanced. This kind of imbalance or nonequilibrium is not simply caused by the difference of the degree of recognition but also by the situation of resources and environmental conditions at various stages. In the long run, it is the result of planning and designing, which is in accord with the characteristics of each stage.

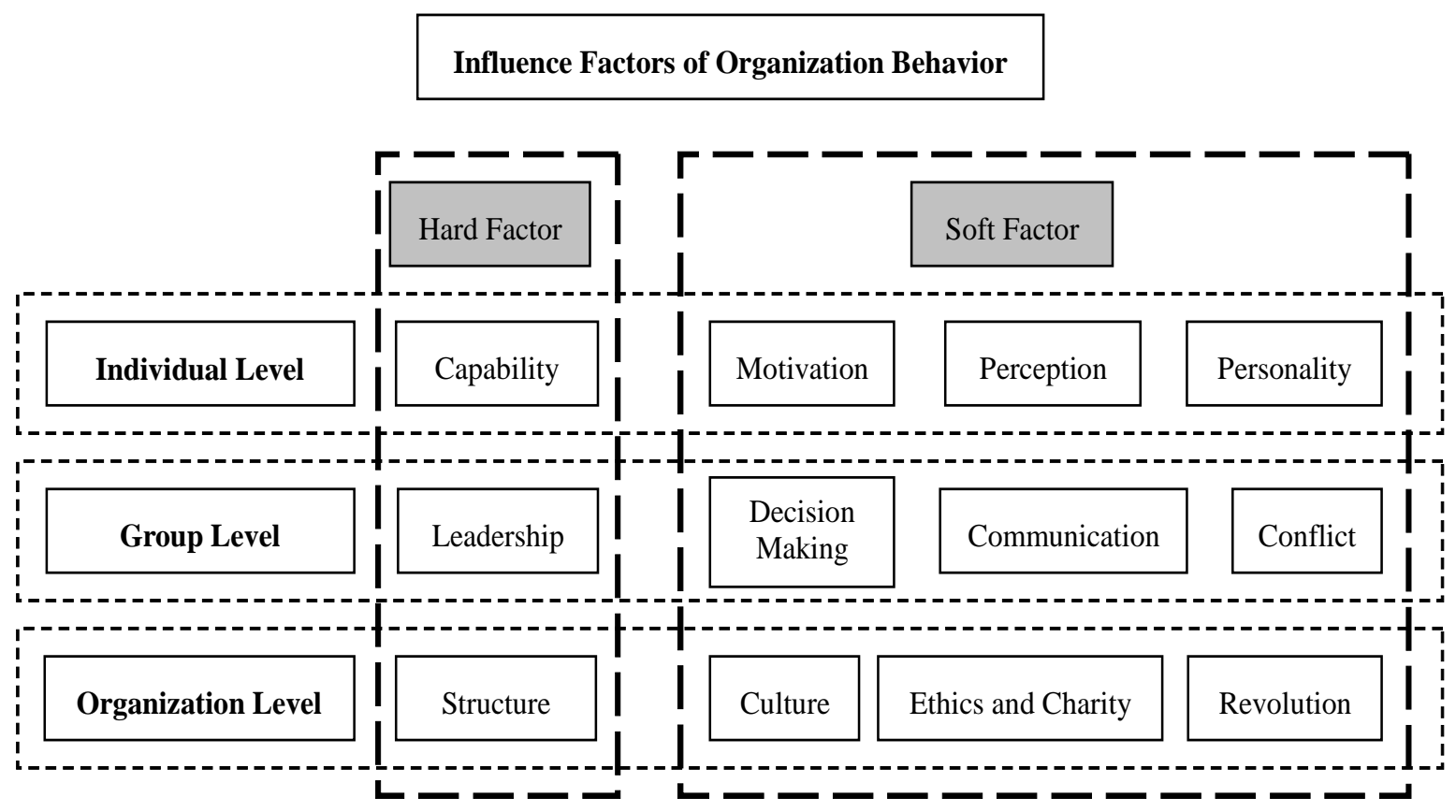

Fig 1. Mainline of the Binary Nonequilibrium

\section{The Paradigm of Organization Behavior Management}

The paradigm of organization behavior management is composed of behavioral structure, behavioral process and behavioral performance. The structure of organizational behavior includes identification, selection and feedback. The identification of organizational behavior is the analysis of the state and manifestation of the organization's internal and external behavior. Its fundamental content involves three aspects which are clarifying the types and forms of various organization 
behavior, justifying the feature and function of concrete action, grasping the development conditions of organization conduct. The selection of organizational behavior is the key to the survival and development of the organization. The selection must be based on two basic essentials, one is the strategic goal of the organization; the other is the external production environment. Organizational behavior feedback refers to the evaluation of its performance under certain circumstances. If the results are satisfied, the current status should be maintained. While if the performance needs to be improved, some measures should be taken to ameliorate organizational behavior and finally enhance the efficiency of the organization. Behavioral process can be unfolded on the basis of the behavioral structure and ultimately reach the behavioral performance of the organization. The behavior structure presents the static elements of organizational behavior management. In different stages, the elements have corresponding influence on the behavior at different levels. These effects will form various organizational behavior processes. The management of organizational behavior will also produce adjustment or change in various organizational behavior process, which thereby yield different results including both economic performance and non-economic performance such as management efficiency.

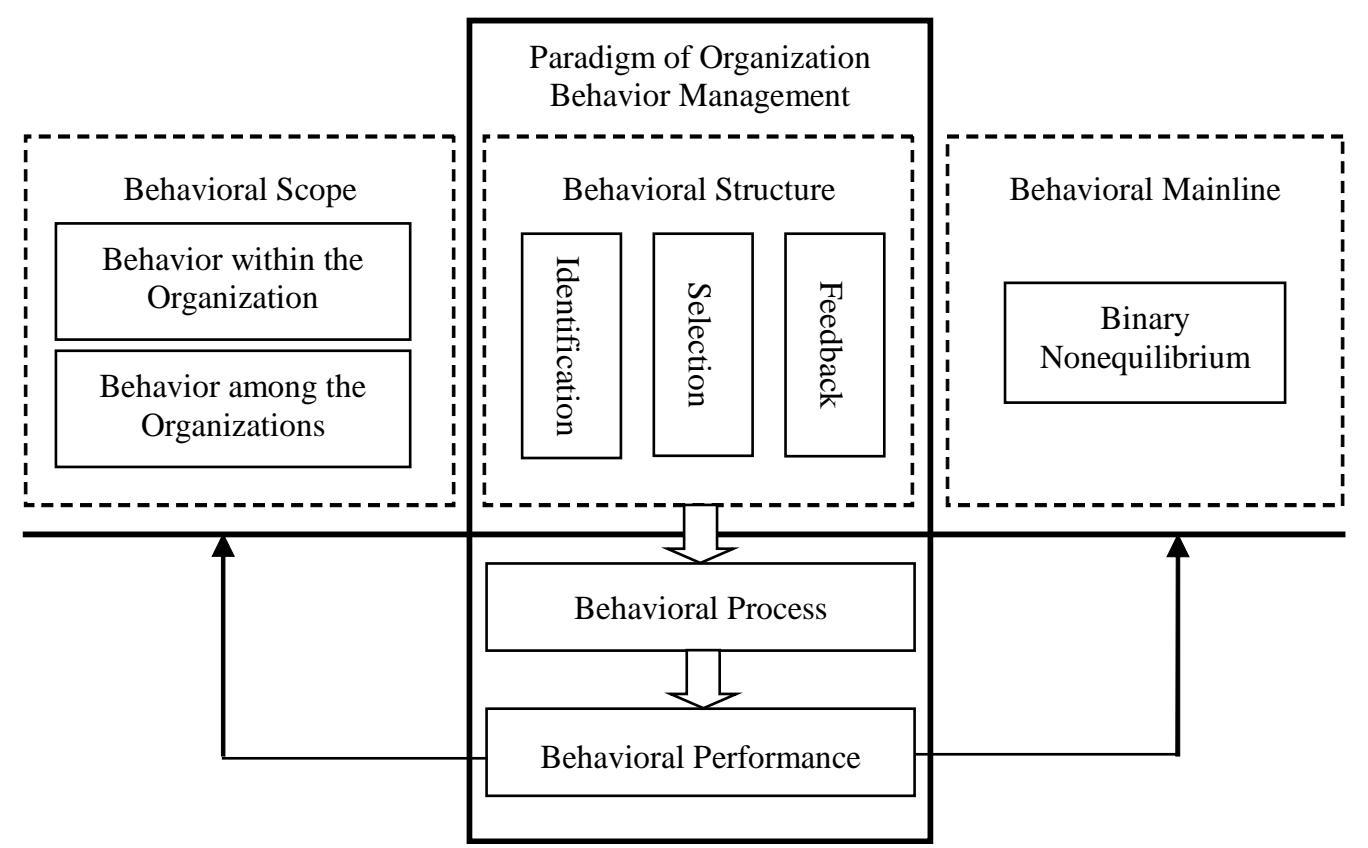

Fig 2. The Paradigm of Organization Behavior Management

The realization of organizational behavior management must clarify the scope and the mainline of behavior. Behavior Scope includes the conducts within the organization and the actions among the organizations. The mainline of binary nonequilibrium clarified the emphases and rules of the development of organizational behavior. At the same time, the scope, structure and mainline of the behavior are interrelated and influence each other. Specifically, the behavioral scope is the basis and condition for the behavioral structure while the behavioral mainline represent the main content and development tendency of the organizational behavior. Further, the behavior structure is the static expression of organizational behavior, and from the dynamic point of view, the factors that influence the behavior of each level determine the organizational behavior process. Behavioral structure and behavioral process together form the organizational behavior performance which generally includes management support, moderate motivation, employee participation, effective communication, scientific assessment, relevant training and standard operation. ${ }^{[2]}$ Finally, the paradigm of the organizational behavior management is constituted of behavioral structure, behavioral process and behavioral performance (see figure 2). 


\section{Acknowledgement}

This research was financially supported by East China University of Political Science and Law under Grant 2017 Science Research Project, Shanghai Social Sciences Planning Project under Grant 2015JG009-BGL307 and the National Social Science Foundation of China under Grant 17BGL099.

\section{References}

[1] KE Jianglin, WU Dan, FAN Liqun. Research Advances in the Measurements, Causes and Effects of Corporate Philanthropic Behavior. Human Resource Development of China, 2014(11):31-37 (In Chinese).

[2] LIU Yaozhong. A Study on the Structure of Key Behavioral Factors of Successfully Implementing performance Management and Its Relationship with Organizational Commitment and Performance. Psychological Science, 2007(4) :967-970 (In Chinese). 